\title{
Stripping extraction of positive ions from a cyclotron
}

\author{
Jasna L. Ristić-Djurović \\ Vinča Institute of Nuclear Sciences, Laboratory of Physics (010), P.O. Box 522, 11001 Belgrade, Yugoslavia
}

(Received 30 August 2001; published 5 December 2001)

\begin{abstract}
In a multipurpose cyclotron, it is convenient to use stripping extraction to extract selected positive ions in addition to the usual extraction by stripping of negative ions. The ions are specified as positive or negative according to the sign of their charge during acceleration. After stripping, positive ions travel not only through the pole edge magnetic field gradient, as is the case with negative ions, but can also pass more than once through regions of large sector edge magnetic field gradients. Influence of the magnetic field gradients on the trajectory of a stripped positive ion and on the extracted beam emittance is studied. It is shown that extraction efficiency depends on the position of the point of beam extraction from a cyclotron and on the ratio between ion charge after and before stripping. The position of the point of extraction also strongly affects the design parameters of the stripping extraction system. Extraction quality and design parameters cannot all be simultaneously optimized. The method of extraction system optimization is suggested and applied to the special case of the VINCY cyclotron.
\end{abstract}

DOI: 10.1103/PhysRevSTAB.4.123501

PACS numbers: 29.27.Ac, 29.20.Hm

\section{INTRODUCTION}

A thin carbon foil can be used to strip some of an ion's electrons and, therefore, in a magnetic field, change the rotation direction of a negative ion or decrease the rotation radius of a positive ion. This method is heavily used for negative ion beam extraction in both commercial and noncommercial cyclotrons [1-3]. The advantages of stripping extraction compared to extraction by electrostatic deflector are the absence of the turn separation requirement as well as the possibility to change the extracted ion energy easily by adjusting the radial position of the stripping foil. The efficiency of stripping extraction applied to a negative ion beam can be as high as $99.9 \%$. A positive heavy ion beam, after interaction with the stripping foil, is distributed among different charge states depending on the ion energy and atomic mass $[4,5]$. Since different charge states are spread in space by the magnetic field, stripping extraction is limited to the part of the beam corresponding to a single charge state. Extraction of positive ions by stripping has several additional disadvantages. After its charge is increased and curvature radius decreased during interaction with the foil, a positive ion executes at least one loop before it leaves the cyclotron. Since the magnetic field of a cyclotron is not designed for such motion, it is likely that the beam quality, measured by beam emittance and envelope, will be disturbed. The disturbances are smaller if acceleration after stripping is avoided. In such a case, the ratio of the ion radii before and after the interaction with the foil, and consequently the ratio of the ion specific charges after and before the stripping, is larger than roughly 2 . Therefore, the charge of a positive heavy ion during acceleration is limited to a maximum of approximately $A / 2$, where $A$ is the ion atomic number. Thus, energy gain per turn is also limited.

\section{DESIGN PARAMETERS AND QUALITY CRITERIA}

The stripping foil extraction system consists of a carbon foil, a mechanism for holding, positioning, and exchange of the foil, and guiding elements used to focus and bend the beam after it interacts with the foil and before it enters a high energy transport line. The guiding elements are usually passive magnetic channels placed inside the cyclotron vacuum chamber or active magnetic elements located outside. For a multipurpose machine, active magnetic elements are a better choice due to their adjustable bending and focusing characteristics. Note that the beam guiding elements of the stripping extraction system may form more than one beam-guiding branch.

Three sets of stripping extraction system parameters are used throughout. The operating parameters define a particular operating regime of the system, design parameters define the system, and performance of the system is measured by the quality parameters. For each operating regime of a cyclotron, corresponding to different ion beams, the operation of a cyclotron is adjusted by setting the values of its operating parameters. The operating parameters of the stripping extraction system are the coordinates of the stripping foil position as well as coil currents of each of the beam guiding elements.

The extraction system should provide extraction not of a single ion beam, but of a group of accelerated beams. The design parameters need to be determined during design of the stripping extraction system and they include the operating area of the stripping foil and parameters of the guiding elements located between the stripping foil and the beginning of the transport line. A beam guiding element is defined by its position, size, maximum bending, and maximum focusing requirements. 
The design of an optimum stripping extraction system is based on the study of system performance and feasibility. Dependence of system performance on the design parameters is obtained by varying key design parameters used as input in beam dynamics simulation. The beam parameters whose ranges of values weigh the extraction system performance and feasibility are the beam losses, emittance, and direction of the extracted beam upon exit from the cyclotron, as well as the corresponding foil position. For a chosen charge state after the foil, the beam losses between the foil and exit from the cyclotron should preferably be zero. The beam emittance upon exit from the cyclotron should be as small as possible since it determines the focusing requirements of the magnetic elements. Also, beam emittance should match the transport line acceptance in order to avoid beam losses along the transport line. The range of directions of the accelerated beams upon exit from the cyclotron sets the bending characteristics of the magnetic elements. These may also be used to decide upon optimum direction of the high-energy transport line. A small gap between sectors may restrict foil position area to the space in the valleys.

\section{METHOD DESCRIPTION}

The proposed method of the stripping extraction system optimization arose from the design of the stripping extraction system for the VINCY cyclotron. The VINCY cyclotron [6] is a multipurpose machine designed to accelerate light ions as well as heavy ions with ion specific charges ranging from $\eta=0.15$ to $\eta=1$. At the extraction radius of $0.86 \mathrm{~m}$, the maximum ion energy is $36 \mathrm{MeV} / n$ for heavy ions and $66 \mathrm{MeV}$ for protons. The cyclotron magnet has four straight sectors per pole, a pole diameter of $2 \mathrm{~m}$, a sector-to-sector gap of $31 \mathrm{~mm}$, and a valley-to-valley gap of $190 \mathrm{~mm}$. The maximum magnetic field in the machine center is $1.97 \mathrm{~T}$.

To study the dependence of beam dynamics on the design parameters of the stripping extraction system, a software package for the beam dynamics simulation was developed [7]. Since expected beam intensities of the VINCY cyclotron are not high enough for space charge effects to be significant, the beam dynamics simulation package uses a charged particle trajectory simulator. The three coupled, nonlinear partial differential equations that describe a charged particle motion are solved using the fourth-order Runge-Kutta method. Calculation of the isochronized magnetic fields in the median plane is based on measured magnetic field maps and performed according to Gordon's procedure [8]. Values of the magnetic fields outside the median plane are determined using $\nabla \cdot B=0$, $\nabla \times B=0$, Taylor's expansion, as well as numerical derivation of the median plane magnetic field. Central difference formulas with the error of order $s^{4}$, where $s$ is the cell size of the measured field grid, are applied to compute derivatives. Values of the magnetic field and its derivatives, at the point in space different from the grid point, are calculated using linear interpolation and values of the magnetic field and its derivatives in the four surrounding grid points.

The stripping extraction system of a multipurpose cyclotron must accommodate an appropriate number of operating regimes corresponding to different ion beams. The number of different operating regimes that must be considered can be reduced using the following argument: A test ion accelerated in a cyclotron to its maximum energy per nucleon $T$ reaches the same maximum designed radius, regardless of its specific charge, $\eta_{b}$. This is achieved by proper scaling between test ion parameters $\eta_{b}$ and $T$ and the parameters of the magnetic field. Nevertheless, the orbits of two different test ions differ. This is because the scaling is valid only for the particular value of the maximum radius while the orbit radius of a test ion is azimuthally dependent. If the current of the main coils is above the iron saturation level, as is the case in acceleration of positive ions within the VINCY cyclotron, the differences among orbits are mainly caused by the trim coils. Since the contribution of the trim coils to the resulting magnetic field is much smaller than the contribution of the main coils, the difference among orbits just before the stripping foil is negligible compared to the size of the stripping foil. Consequently, it is sufficient to study behavior of a single ion beam, defined by a single set of values of the parameters $\eta_{b}$ and $T$.

Two different ion beams will continue to behave the same after foil if their $K$ parameters are the same, where $K$ is the ratio of the ion specific charge after and before the foil, namely $K=\eta_{a} / \eta_{b}$. The condition $K \geq 2$ has to be satisfied in order to avoid ion acceleration after interaction with the foil. Consequently, the largest range of $K$, corresponding to the largest variety of ion orbits after interaction with the foil, is obtained for the smallest $\eta_{b}$. So, to study stripping extraction of all positive ions that can be extracted using this method, it is sufficient to study the extraction of an ion with the smallest specific charge before the foil. For the VINCY cyclotron, the smallest value of $\eta_{b}$ is 0.15 and the largest corresponding ion energy is $3.1 \mathrm{MeV} / n$. It was assumed that the stripping foil is positioned along the equilibrium orbit of this ion and beam behavior is studied for different values of $K$.

\section{INFLUENCES OF MAGNETIC FIELD GRADIENTS}

Because of the loop(s) that a positive beam executes after stripping, beam dynamics is strongly influenced by the magnetic field gradient not only at the pole edge but also at sector edges. At the pole edge the beam is radially defocused. The sector edge gradients can cause both drift and (de)focusing of the beam. 


\section{A. Drift}

Inward and outward drifts of a test ion, caused by the sector edge gradients, are shown in Fig. 1. The outward drift helps extraction since it pulls the ion away from the cyclotron center. The inward drift makes extraction more difficult and also less efficient as the emittance of the beam that executes the multiple loops is expected to be large.

The position of the stripping foil on the equilibrium orbit and the $K$ parameter determine the number of loops after foil as well as the point of exit of the ion from the cyclotron. The magnetic field symmetry narrows the range of the azimuthal foil positions that should be considered to the angle between the axes of the two neighboring sectors. In our four sectors per pole example this range is $\theta_{f} \in\left[0^{\circ}, 90^{\circ}\right]$. Figure 2 shows the dependence of the exit point on the foil position. Successive "hops" on $\theta_{e}\left(\theta_{f}\right)$ curves are caused by the sector edge gradients and correspond to ion trajectories with one, two, or three loops after the foil. Figure 3 illustrates all three types of trajectories

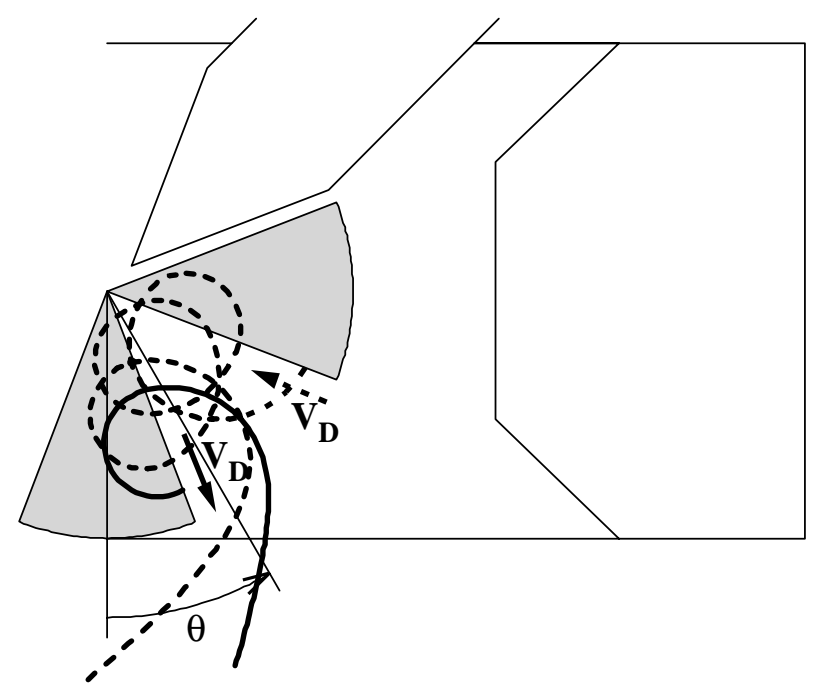

FIG. 1. Drifts caused by magnetic field gradients. The example shown corresponds to the magnetic field of the VINCY cyclotron and a test ion with $K=3$. If the foil is positioned in the region where the ion, along its equilibrium orbit, travels from a lower to a higher magnetic field, ion drift after the foil is directioned outwards. Ion trajectory and corresponding drift direction are depicted by a solid line. If the foil is positioned in the region of a negative magnetic field gradient corresponding to the sector-valley pass, ion drift after the foil is directioned towards the cyclotron center and the ion executes multiple loops, as represented by the dotted line. If the gap between sectors is not large, multiple loops cause beam losses. Also shown is the zero and positive direction of the azimuthal coordinate used further throughout the paper. Because of axial symmetry, only half of the magnet yoke and one of the two rf cavities of the VINCY cyclotron are sketched. Extraction area is limited to $\theta \in\left[-20^{\circ}, 65^{\circ}\right]$. for a test ion with $K=3.33$. The number, position, and width of the hops on $\theta_{e}\left(\theta_{f}\right)$ curves depend on the $K$ parameter and the sector geometry (number of sectors per pole, azimuthal width of a sector, sector type - straight or spiral, etc.).

As seen in Fig. 2, in the VINCY cyclotron an ion can be brought to a chosen exit point by as many as six different stripping foil positions corresponding to a single, double, or triple loop trajectory. However, ion beams with multiple loop trajectories are expected to suffer larger disturbances in terms of their envelopes and emittances. Therefore, only single loop trajectories will be further discussed. Since all curves $\theta_{e}\left(\theta_{f}\right)$ pass through the vicinity of the point $\left(\theta_{f}, \theta_{e}\right)=\left(47.5^{\circ}, 3.5^{\circ}\right)$, the exit point $\theta_{e}=3.5^{\circ}$ seems to be optimal in terms of the foil position range as it requires the smallest stripping foil operating area. Unfortunately, this point is also very close to the transition of a single to double loop trajectory. Therefore, the beam envelope disturbances are to be expected and the extraction point $\theta_{e}=3.5^{\circ}$ is likely to be far from optimal in terms of beam quality.

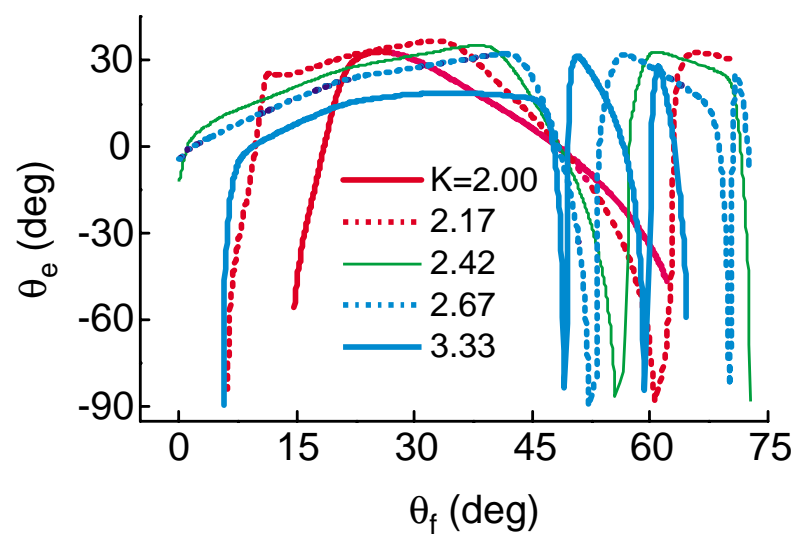

FIG. 2. (Color) Exit point dependence on foil position. The radius of the ion exit from the vacuum chamber of the VINCY cyclotron is $1.6 \mathrm{~m}$. The exit point is defined by its azimuthal coordinate $\theta_{e}$ along the exit radius. Foil position is also defined by its azimuthal coordinate $\theta_{f}$, but along the equilibrium orbit of an ion. The $\theta_{e}$ dependence on $\theta_{f}$ is strongly influenced by the $K$ parameter. The "hops" that $\theta_{e}$ exhibits during $\theta_{f}$ increase correspond consequently to a single, double, and triple loop trajectory of a stripped ion (see Fig. 3). If the loop radius is small, i.e., if $K$ is large, the $\theta_{e}\left(\theta_{f}\right)$ curve exhibits three hops, and an ion trajectory can have as many as three loops after the foil (see $K=3.33$ and 2.67). The larger the loop radius, the smaller the maximum number of loops. The transition to a different number of loops in a trajectory is caused by inward or outward drift. These transitions, for $K=3.33$, are illustrated and explained in Fig. 3 . Note the width $\Delta \theta_{f}$, and the maximum $\theta_{e}$, of the hops corresponding to a one loop extraction. For ion energies lower than the largest, the width and the maximum of the hop is smaller. For different cyclotrons, the curve parameter $K$ should be replaced with $K / V$, where $V$ is the angular width of the valley. For the VINCY cyclotron, $V=48^{\circ}$. 

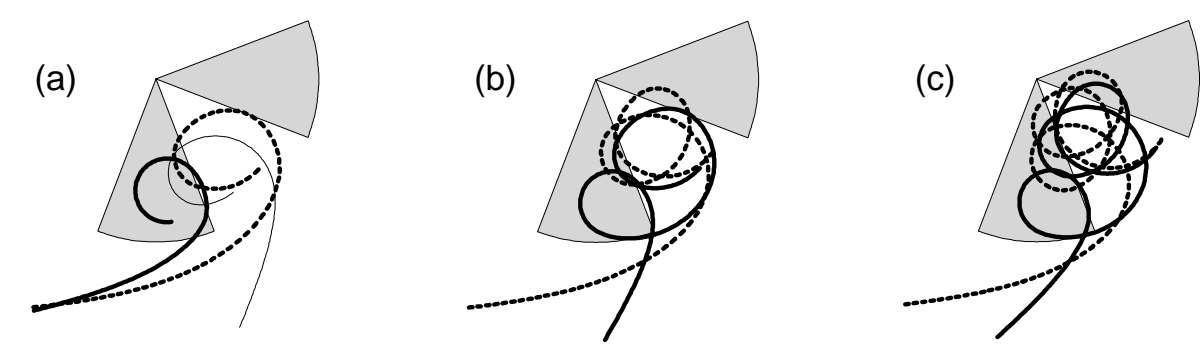

FIG. 3. Single, double, and triple loop trajectories. Sample trajectories of a test ion with $K=3.33$ and with one, two, and three loops after the foil are shown in plots (a), (b), and (c), respectively. Trajectories in plot (a) correspond to the three foil positions on the first hop of the $\theta_{e}\left(\theta_{f}\right)$ curve with $K=3.33$ in Fig. 2. The trajectory represented by a thick solid line illustrates the behavior around the lower limit of the $\theta_{f}$ range of a one loop trajectory existence. If the foil is moved to lower azimuthal angles, influence of the outward drift disappears; the loop, due to its small radius, fits completely over the sector and the ion makes as many as eight loops before exiting the cyclotron. The ion whose trajectory is marked with a dashed line begins the transition from a one loop to a two loop trajectory. If the stripping foil is moved to larger $\theta_{f}$, the first loop crosses the inward drift sector edge, as shown by the thick solid line in plot (b). The sector edge gradient pulls the ion inside, and the ion conducts one more loop. Further increase of $\theta_{f}$ causes both the first and the second loop to move inwards, as illustrated by the double loop trajectory depicted by a dashed line in plot (b). When the second loop is shifted enough inwards, the double loop trajectory, indicated by a dashed line in plot (b), transforms to the triple loop trajectory indicated by a solid line in plot (c).

\section{B. Defocusing}

When a beam travels through a magnetic field gradient perpendicularly to the gradient, the result is the beam defocusing along one and focusing along the other of the two beam axes. The beam axes travel with the beam and are perpendicular to the velocity of the central ion. Since beam orbit is confined to the median plane of a cyclotron, one beam axis is chosen to be aimed verti- cally, the $z$ axis, and the other aimed horizontally, the $h$ axis. Figures 4 and 5 illustrate focusing and defocusing effects of magnetic field gradients. Figure 4 is devoted to sector edge effects, while Fig. 5 compares the influences of various magnetic field gradients. The beam envelopes are affected mostly by sector edge gradients. Influences of other magnetic field gradients are much smaller if not combined with the influence of the sector edge gradient.
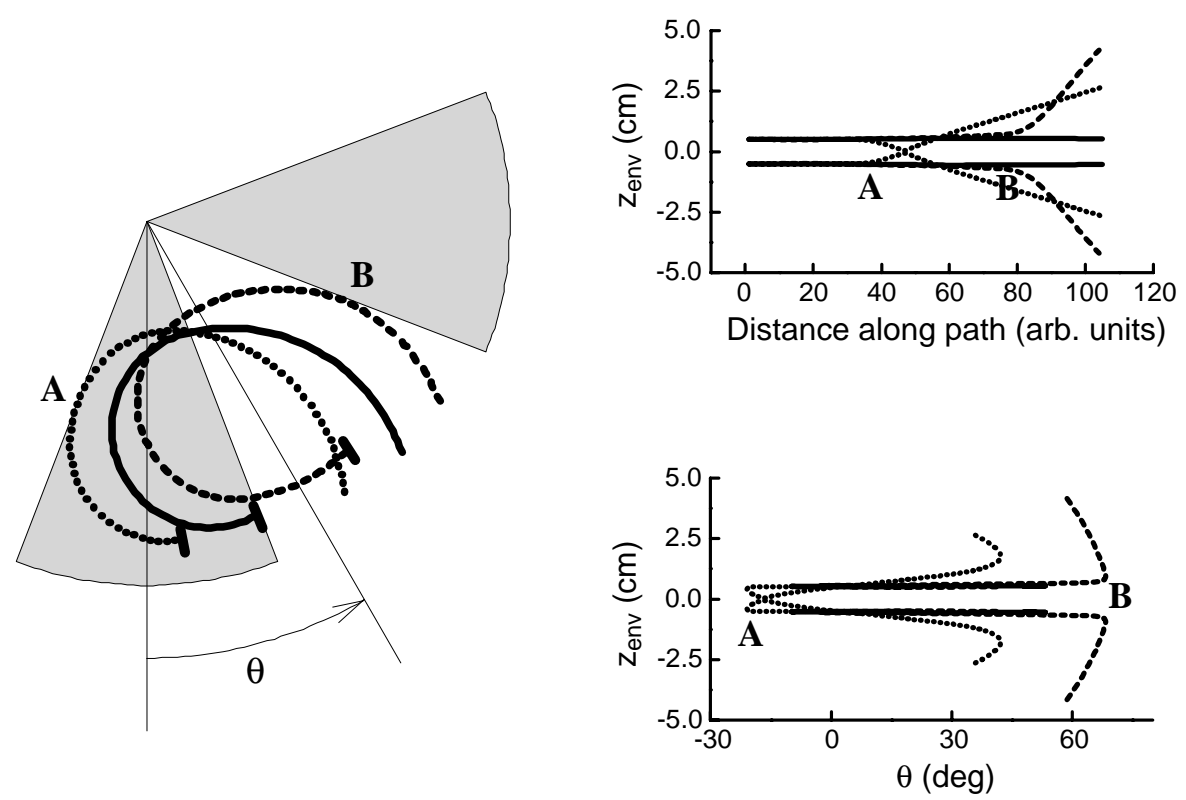

FIG. 4. Influence of the sector edge magnetic field gradient on the beam envelope. The left-hand plot shows three trajectories of test ions with $K=2.42$. These are the central ions for test beams whose radial (horizontal) and axial (vertical) emittances at the foil position are taken to be $\varepsilon_{h}=2 \mathrm{~mm} 6 \mathrm{mrad} \pi=12 \pi \mathrm{mmmrad}$ and $\varepsilon_{z}=5 \mathrm{~mm} 1 \mathrm{mrad} \pi=5 \pi$ mm mrad, respectively. The energy spread of the beam is assumed to be $\pm 1 \%$. The two graphs on the right show the axial beam envelope along the beam path (upper graph) and with respect to the azimuthal position (lower graph). If the beam does not pass close to the sector edge its envelope does not suffer any visible changes, as represented by the solid line. Sector edge B causes defocusing in the axial direction of beam depicted by a dashed line. The beam shown by a dotted line is axially focused by the sector edge A and further over focused along the path. 

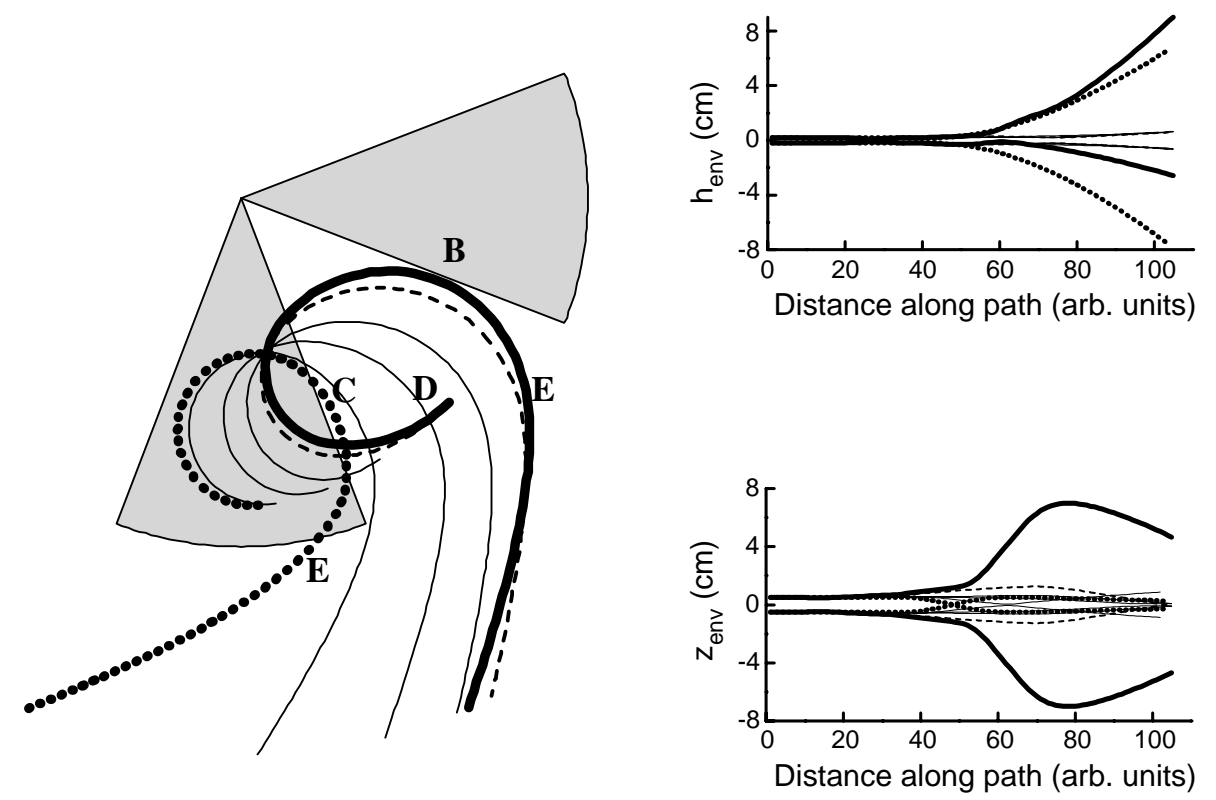

FIG. 5. Influence of different magnetic field gradients on beam envelopes. Extraction of a beam with $K=3$ is studied. Test ions whose trajectories are shown on the left-hand plot are used as the central ions for corresponding test beams. Initial beam emittances and energy spread are taken to be the same as for Fig. 4. The two graphs on the right show horizontal (upper graph) and axial (lower graph) envelopes of the beam along the beam path. Envelopes are calculated with respect to the central ion in the beam. Influences of sector edge gradients in regions $\mathrm{C}$ and $\mathrm{B}$, the pole edge gradient in the $\mathrm{E}$ region, and the isochronous increase of the magnetic field in the D region are investigated. Thin solid lines show trajectories and corresponding envelopes of the beams influenced only by the pole edge gradient. This influence is the least pronounced. The joint effect of the gradients in regions D and E is also small, as shown by a thin dashed line. When the weak influence of region $\mathrm{E}$ is combined with sector edge influence (region $\mathrm{C}$ ), the resulting effect is strong, as represented by a dotted line. Finally, a thick solid line is used to show the combined effect of the D, B, and E regions. The dramatic difference in beam envelopes represented by thick solid and dashed lines is caused by sector edge $\mathrm{B}$, while sector edge $\mathrm{C}$ is the cause of the difference between the examples depicted by thin solid and dotted lines.

When the foil is moved from $\theta_{f}=90^{\circ}$ in the negative $\theta$ direction, the beam first experiences inward drift along the sector edge B (see Fig. 1). Then the dominant effect becomes defocusing of the $z$ envelope by the same sec- tor edge along the outward portion of the beam loop (see Fig. 4). When the azimuthal position of the foil is sufficiently lowered, the beam crosses the sector edge $\mathrm{C}$ and a positive influence of the outward drift takes effect (see
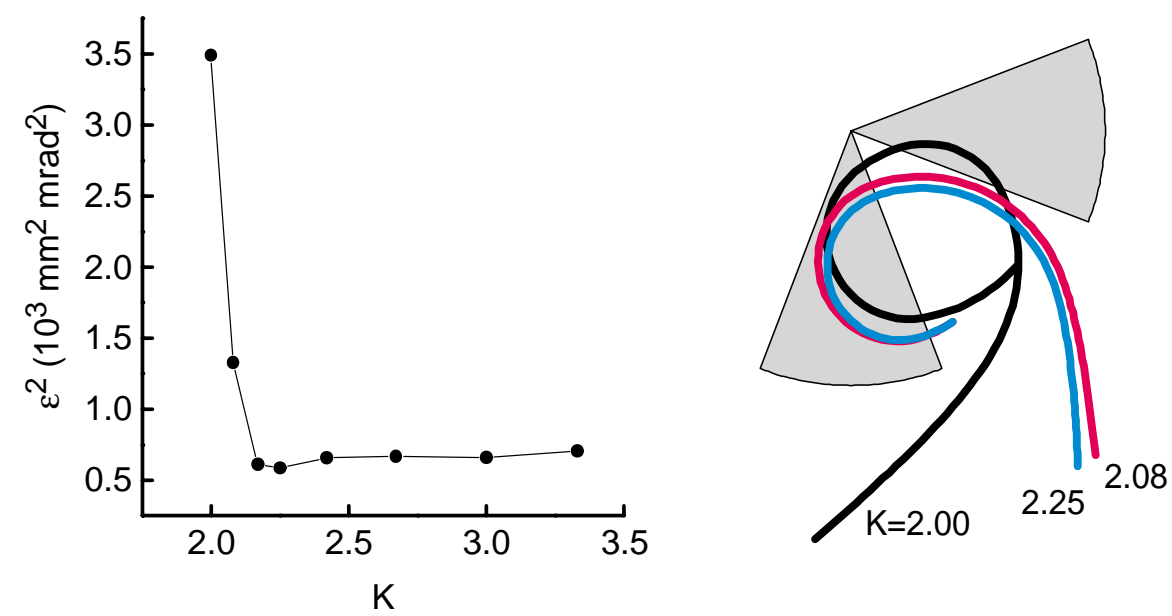

FIG. 6. (Color) Dependence of minimum square emittance on the $K$ parameter. Behavior of the beams with initial emittances and energy spread as in Fig. 4, and with foil positions along the equilibrium orbit, is simulated. Beams with minimum square emittances at the exit from the cyclotron are represented as points on the left graph. Minimum square emittances for $K \geq 2.17$ do not depend much on $K$ and are of the order of $600 \mathrm{~mm}^{2} \mathrm{mrad}^{2}$. Central trajectories of beams with minimum square emittance shown in the right-hand plot justify larger values of the square emittance for $K=2$ and 2.08 . For $K=2.08$, the beam passes close enough to the sector edges both on the inward and outward portion of the loop to experience defocusing. For $K=2$, the radius of the loop is so large that, in addition to defocusing, a small inward drift is present. 
Fig. 1). Finally, at low $\theta$, the dominant negative influence on the beam with a small or large value of $K$ comes from sector edges A or C, respectively (see Figs. 4 and 5). The foil should be positioned in such a way to avoid the negative (defocusing and inward drift) as well as to take advantage of the positive (outward drift) influence of the sector edge. Therefore, the beam loop should cross the sector edge causing outward drift, and the inward and outward portions of the beam loop should be away from the sector edges. For larger values of $K$, the radius of the beam loop is small, therefore, the loop is easier to distance from the defocusing sector edges, and the range of the foil positions resulting in small beam emittance is larger. For a small $K$, the loop radius may be too large to avoid defocusing sector edges.

As a measure of beam quality, square emittance is defined as $\varepsilon^{2}=\varepsilon_{h} \varepsilon_{z}$, where $\varepsilon_{h}$ and $\varepsilon_{z}$ are beam emittances in the horizontal and axial directions, respectively. Emittances $\varepsilon_{h}$ and $\varepsilon_{z}$ correspond to ellipses which enfold not less than $95 \%$ of the distorted phase ellipses obtained by beam simulation. Dependence of the minimum exit square emittance on the $K$ parameter is shown in the left-hand graph of Fig. 6. Foil positions corresponding to minimum square emittance appear in the valley. Exit points of the optimum beams differ significantly and depend on $K$, as can be seen from the three sample central trajectories shown in the right-hand plot of Fig. 6.

\section{RESULTS}

From Fig. 6 it can also be concluded that there is no such position of the first guiding element (the beam exit point from the cyclotron) that will grant minimum exit emittance for all beams with different $K$. Therefore, the best extraction system is to be found through an optimization procedure.

The optimization procedure is performed in four steps. First, extraction system quality parameters are ranked by their importance. Then, dependence of the quality parameters on the key design parameter(s) is studied. These studies are then used to find the optimum key design parameter(s). Finally, the remaining design parameters, other than the key design parameter(s), are determined. Investigating the influence of design parameters on quality parameters and establishing the key design parameter(s) are not easy tasks because of various connections and overlapping between the design and quality parameters. For instance, foil position is a point in the operating area of the stripping foil holder (design parameter), but whether or not it is over sector can be a question of quality. The maximum focusing requirement of guiding elements (design parameter) is defined by maximum exit emittance (quality parameter). The smaller the maximum bending requirements of the guiding elements (design parameters) the better, and these are set by beam exit directions (quality parameters) as well as transport line direction.
The exit point from the cyclotron $\theta_{e}$ was chosen as the key design parameter. This is not only because all quality parameters depend on $\theta_{e}$, but also because all design parameters can be viewed as functions of $\theta_{e}$.

The most important task, in terms of extraction quality, was to avoid beam losses. Consequently, only beams executing a single loop were considered, since all multiple loop beams suffer losses after stripping. The remaining quality parameters of a test beam are ranked as follows: square emittance $\varepsilon^{2}$ being the first, beam direction at the exit point $\varphi_{d}$ as the second, and third, and least importantly, the indicator if the corresponding foil position happens over a sector. It was assumed that the beams that were to be accelerated and extracted could have any value of $K$ within the range $2 \leq K \leq 3.33$.

The dependence of beam square emittance on the exit point is shown in Fig. 7. To extract beams with $\varepsilon^{2}$ as small as possible for all values of $K$, two extraction branches are needed. An extraction point of $\theta_{e}=\theta_{E 1}=31^{\circ}$ could be used to extract beams with $2.17 \leq K \leq 2.67$ and

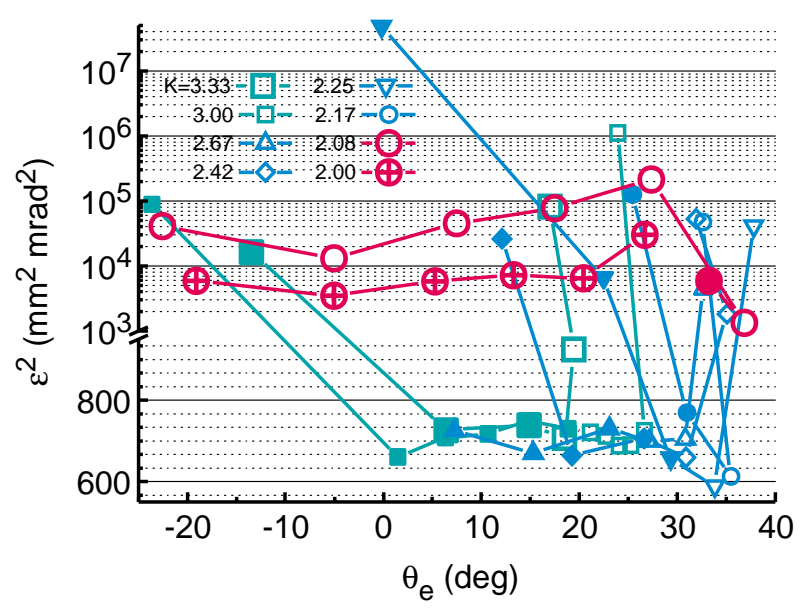

FIG. 7. (Color) Dependence of square emittance on the exit point. For $K=2$ and 2.08, the radius of the loop after the foil is large and defocusing along sector edges cannot be avoided. Corresponding square emittances are 2 orders of magnitude larger than those of beams with larger $K$. For $K \geq 2.17$, the width of the region where $\varepsilon^{2}$ is small increases with $K$, as expected. Values of square emittance between sudden increases are of the order of $700 \mathrm{~mm}^{2} \mathrm{mrad}^{2}$ and do not depend much on the extraction point nor the $K$ parameter. Sudden increases of the order of magnitude of square emittance correspond to negative influences of the magnetic field gradients. The upper $\theta_{e}$ limits of low $\varepsilon^{2}$ segments are set by the sector edge B (Figs. 4 and 5). The lower $\theta_{e}$ limits of low $\varepsilon^{2}$ segments for $2.17 \leq K \leq 2.67$ and $K \geq 3$ are set by sector edges $\mathrm{A}$ and $\mathrm{C}$, respectively (Figs. 4 and 5). Solid symbols on data points depict less favorable foil positions placed over a sector, while open symbols correspond to foil positions in the valley. For another cyclotron, the curve parameter $K$ should be replaced with $K / V$, where $V$ is the angular width of the valley. Consequently, for a broader valley, e.g., a three sector per pole cyclotron, the problem of large emittance of the beams with $K \leq 2.08$ may disappear. The square emittance of negative ion beams is not shown because it is practically independent of $\theta_{e}$ and is smaller than the square emittance of positive ion beams. 
optionally $K=2.08$. A second extraction point of $\theta=\theta_{E 2}$ would be used for $K=2.00,3.00,3.33$, and optionally 2.08. Small $\varepsilon^{2}$ for $K \geq 3.00$ would be provided by $\theta_{E 2}=5^{\circ}$, while $\theta_{E 2}=-5^{\circ}$ would provide minimum extracted $\varepsilon^{2}$ for $K=2$. Foil positioning in the small gap over sector is inevitable regardless of chosen exit points. Note that a large value of $\theta_{E 1}$ limits the range of extracted ion energy (see the caption of Fig. 2).

Selected exit points establish some design parameters. The exit points are also the entrances of the guiding elements which beams encounter first along the extraction branches. The operating area of the stripping foil is defined using Fig. 2, extended to include curves for all values of $K$. Only those portions of curves that correspond to foil positions used for the beam dynamics simulations represented in Fig. 7 are considered. For a particular $K$, this provides uniqueness of the stripping foil position corresponding to a chosen exit point. To determine maximum focusing requirements, the beam that is most difficult to focus has to be considered for each of the extraction branches. In our example these are the beams with $K=2$ and 2.08. The necessary focusing gradients of the two guiding elements are found through a number of beam dynamics simulations until beam emittances at the beginning of the transport line match transport line acceptance as close as possible.

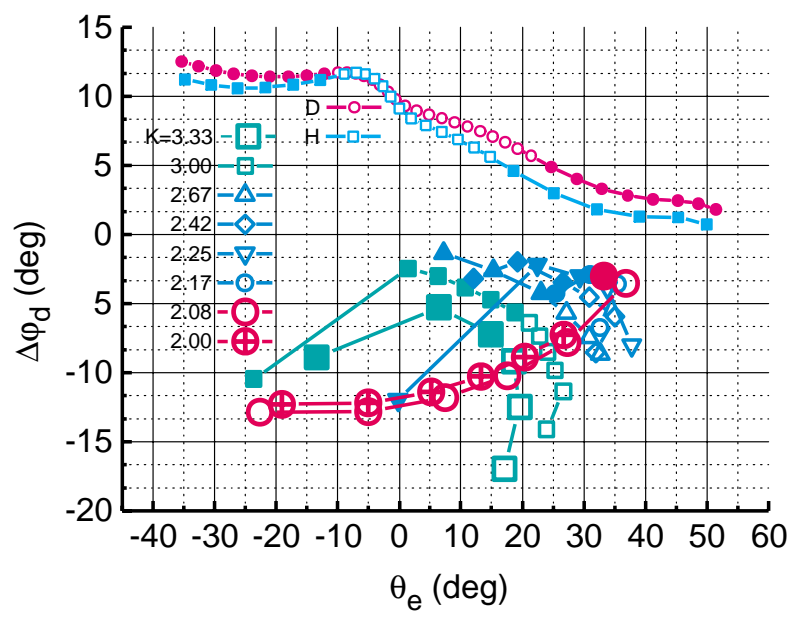

FIG. 8. (Color) Dependence of beam direction on its exit point. To measure a beam direction angle, $\varphi_{d}$, the origin of the coordinate system in Figs. 1 and 4 is translated from the cyclotron center to a beam exit point. The results are expressed as $\Delta \varphi_{d}=\varphi_{d}-\left(1.125 \theta_{e}-31.25\right)$ in order to make the difference between curves corresponding to different $K$ more pronounced. Unlike $\varepsilon^{2}$, the direction angle of a negative ion beam $\left(\mathrm{D}^{-}\right.$and $\left.\mathrm{H}^{-}\right)$depends on $\theta_{e}$ and therefore must be considered during extraction system optimization. For positive ion beams, the represented data points correspond to the same set of test beams whose values of $\varepsilon^{2}$ are shown in Fig. 7. As in Fig. 7, solid symbols on data points depict beams with foil position placed over a sector, while open symbols correspond to foil positions in the valley. The smaller the angular spread of beams in an exit point, the narrower the corresponding range of the bending magnetic field necessary for the adjustment of beam directions to the transport line direction.
If the direction of the transport line is set as a requirement, the differences between transport line direction and beam directions at the exit point are used as quality criterion. However, if possible, it is better to use beam directions to determine the optimum direction of the transport line. Beam direction dependence on the exit point is shown in Fig. 8. If all beams with $2 \leq K \leq 3.33$ are to be extracted, angular spread of the beams is narrowest at $\theta_{e} \cong 18^{\circ}$. However, at this exit point, square emittances are very large for $K \leq 2.25$ (see Fig. 7).

The best sequence of steps, during design of the stripping foil extraction system, is to first optimize the position of the exit point with respect to beam emittance and, if necessary, foil position range, and then to use beam directions to optimize direction of the transport line. Transport line direction is optimized by minimizing the required range of the bending magnetic field. This is performed using the equation $B_{\text {bend }}=\operatorname{const} \Delta \varphi_{d} / K$, where $B_{\text {bend }}$ is the required bending field and $\Delta \varphi_{d}$ is the difference

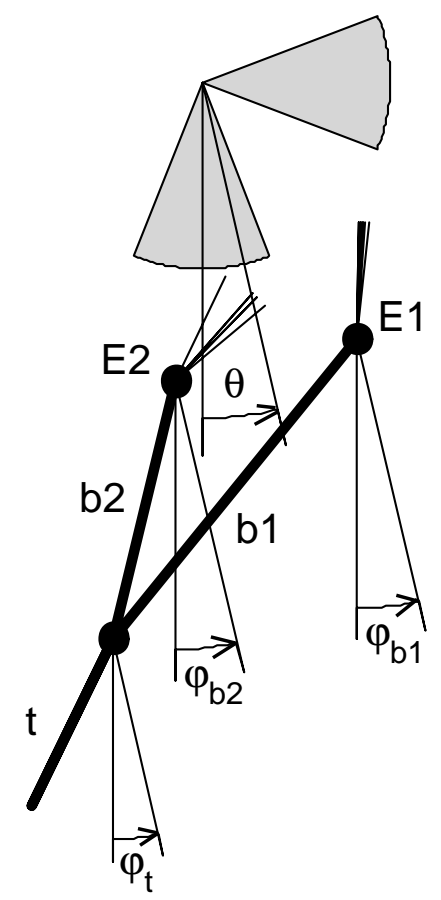

FIG. 9. Optimum direction of the transport line and extraction branches. Optimization was performed for the extraction with two exit points. Positive ion beams with $2.08 \leq K \leq 2.67$ are extracted through the exit point $E 1$ whose $\theta_{E 1}=31^{\circ}$. Negative ion beams of $\mathrm{H}$ and $\mathrm{D}$, and positive ion beams with $K=2.00$ and $K \geq 3.00$ are extracted through the exit point $E 2$ whose $\theta_{E 2}=-5^{\circ}$. Short thin lines passing through an exit point depict incoming test beam directions. Thick lines represent optimum directions of the two extraction branches and transport line. It was assumed that the two extraction branches cross at $R=3 \mathrm{~m}$. Optimum beam transport directions require the narrowest range of the bending magnetic field. Obtained values of the first and second extraction branch direction and transport line direction are $\varphi_{b 1}=-39^{\circ}, \varphi_{b 2}=-14^{\circ}$, and $\varphi_{t}=-26^{\circ}$, respectively. The expected maximum required bending magnetic field is approximately $0.5 / L_{m} \mathrm{~T}$, where $L_{M}$ is the length of the bending magnet in $\mathrm{m}$. 
between beam direction and transport line direction. This procedure is more complicated if there are two extraction branches. In that case, these two branches cross at the beginning of the transport line. A guiding element is placed in each of the two exit points as well as at the beginning of the transport line. Optimum directions of the branches and the transport line are determined by minimizing the maximum of three magnetic field ranges corresponding to these three guiding elements; see Fig. 9.

It should be noted that maximum bending requirements of the guiding elements are also determined by the described line direction optimization. The obtained line directions define the coordinates of guiding elements other than $\theta_{E 1}$ and $\theta_{E 2}$.

\section{CONCLUSION}

In a multipurpose machine, it is useful to extend stripping extraction, commonly applied to negative ions, to include positive ions. Thus stripping extraction of positive ions has been studied in detail. The rotation radius of a positive ion is decreased after stripping and before exiting the cyclotron, this ion executes at least one loop. Therefore, since sector edge gradients significantly influence the dynamics of positive ion beams, this influence is carefully considered in order to achieve efficient extraction. It was shown that the defocusing effect of the magnetic field gradient, occurring when the beam travels along the sector edge, should be avoided. Inward drift should also be avoided because it causes extraction to be less efficient and harder to achieve, while advantage should be taken of outward drift. For efficient extraction, only beams with a single loop are to be considered.

A particular solution for an extraction system is based on the study of the dependence of beam quality parameters (beam losses, square emittance, and direction of the beam in the exit point as well as stripping foil position) on system design parameters (operating area of the stripping foil, positions, sizes, maximum bending, and maximum focusing requirements of the guiding elements). This study is simplified if the key design parameter used as an argument in the presentation of quality versus design parameters is properly chosen. As the key design parameter, the beam's exit point from the cyclotron $\theta_{e}$ is used. For a chosen design of the extraction system, $\theta_{e}$ is fixed, since it coincides with the position of the first guiding element. Also all quality and other design parameters can be represented as functions of $\theta_{e}$.

It was shown then, that for a large enough range of $K$, it is impossible to optimize even a single quality parameter (e.g., exit emittance or foil position) for all desired beams. Therefore, during the extraction system design, a trade-off between achieved quality parameters is inevitable. For example, large values of $\theta_{e}$ enable efficient extraction of beams from the broader range of their $K$ parameter (see Fig. 7), but also increase the lower limit of extracted ion energy (see Fig. 2). In our example, the lower limit of extracted energy was not considered as a quality parameter. However, the chosen key design parameter enables broadening of the study to include the lower energy limit. The analysis of dependence of quality parameters on the key design parameter (Figs. 2, 6, and 7) should be extended to include curves corresponding to different extraction energies. Each curve will then be characterized by two parameters: the $K$ parameter as well as the extraction energy. In general, quality parameters should be carefully defined and ranked in order to ease comparison of possible extraction system realizations.

The suggested method of the stripping extraction system design can be divided into four steps. First, square emittance, beam direction, and foil position should be represented as functions of $\theta_{e}$, using $K$ and, eventually, ion extraction energy as parameters. If there are any restrictions on $\theta_{e}$ or the stripping foil position, caused by geometry and/or lower ion energy limit requirements, the portions of the curves corresponding to the restricted regions should be disregarded. As a second step, the optimum value of $\theta_{e}$ is chosen with respect to square emittance. This will minimize beam losses as well as focusing requirements for beam guiding elements. Optimum directions of the transport line and extraction branch(es) should be considered in the third step. Optimum directions are found by minimizing corresponding bending requirements of the guiding elements for the chosen exit point. In addition, these directions, together with the exit point position, set placement of the guiding elements. Finally, the operating area of the stripping foil is read from foil position dependence on $\theta_{e}$, and the apertures of the guiding elements are set according to maximum beam envelopes.

[1] V Bechtold, in Proceedings of the 13th International Conference on Cyclotrons and their Applications, Vancouver, Canada, 1992, edited by G. Dutto and M. K. Craddock (World Scientific, Singapore, 1993), p. 110.

[2] G. Dutto, in Proceedings of the 13th International Conference on Cyclotrons and their Applications, Vancouver, Canada, 1992 (Ref. [1]), p. 138.

[3] Y. Jongen et al., in Proceedings of the 11th International Conference on Cyclotrons and their Applications, Tokyo, 1986, edited by M. Sekiguchi, Y. Yano, and K. Hatanaka (Ionics Publishing, Tokyo, 1987), p. 275.

[4] E. Baron and B. Delaunay, Phys. Rev. A 12, 40 (1975).

[5] K. Shima, T. Ishihara, and T. Mikumo, Nucl. Instrum. Methods Phys. Res. 200, 605 (1982).

[6] N. Nešković et al., in Proceedings of the 14th International Conference on Cyclotrons and their Applications, Cape Town, 1995, edited by J.C. Cornell (World Scientific, Singapore, 1996), p. 82.

[7] J. Ristić-Djurović, N. Nešković, and S. Ćirković, in Proceedings of the 6th International Computational Accelerator Physics Conference, Darmstadt, 2000, http://www. icap2000.de/

[8] S. Ćirković, MS thesis, Belgrade University, 2001. 